\title{
Editorial \\ Plasma gelsolin as a biomarker of inflammation
} Mark J DiNubile

Division of Vaccines/Infectious Diseases, Medical Communications Department, Merck Research Laboratories, PO Box 1000, UG3C-06, North Wales, PA 19454-1099, USA

Corresponding author: Mark J DiNubile, mark_dinubile@merck.com

Published: 3 December 2008

Arthritis Research \& Therapy 2008, 10:124 (doi:10.1186/ar2547)

This article is online at http://arthritis-research.com/content/10/6/124

(c) 2008 BioMed Central Ltd

See related research article by Osborn et al., http://arthritis-research.com/content/10/5/R117

\begin{abstract}
The prognostic implications of declining plasma gelsolin levels have been documented after a diverse variety of acute insults. Because gelsolin concentrations fall prior to the development of complications, a pathophysiological role for gelsolin depletion has been postulated in delayed multiorgan failure. The original hypothesis about the function of circulating gelsolin was that it scavenged actin released from cells at the site of injury. Although extracellular actin may be the primary cause of gelsolin depletion, the biologic imperative for gelsolin could entail the modulation of several inflammatory mediators as much as the disposal of actin. Translational research is actively addressing whether replenishment of plasma gelsolin could provide an efficacious and well tolerated therapeutic intervention in selected seriously ill patients.
\end{abstract}

Osborn and colleagues demonstrate that patients with rheumatoid arthritis and knee effusions have plasma gelsolin levels lower than those in healthy volunteers [1]. Gelsolin was first identified as a cytoplasmic actin-regulatory protein essential for cell locomotion and phagocytosis. When a circulating isoform was subsequently discovered, its physiological significance was far from apparent.

For over two decades, researchers have struggled to define the raison d'être for actin-binding proteins in plasma, which under normal circumstances does not contain detectable actin. A straightforward hypothesis emerged that plasma gelsolin scavenges actin leaked into the circulation or interstitial space from injured tissue in order to abort subsequent damage instigated by extracellular actin filaments [2]. Hypogelsolinemia ensues after a wide spectrum of seemingly unrelated types of insults and precedes a second wave of injury that typically involves organs distant from the precipitating event [2-5]. The accumulated phenomenology has given way to plausible mechanisms, but rigorous science has yet to fully catch up with vigorous speculation.

Current dogma has expanded to now accommodate the convincing observations that plasma gelsolin can bind a variety of potentially inflammatory moieties, and can thereby modulate the exuberance of the host response to sepsis, malaria, burns, trauma, and other commonly encountered clinical conditions. Plasma gelsolin's primary function may be to protect seriously ill patients from their own overzealous inflammatory response. Identified ligands include plateletactivating factor, lysophosphatidic acid, and sphingosine 1 phosphate, as well as cell-wall constituents of Gram-negative and Gram-positive bacteria [6-8]. Actin exposed at the site of overwhelming injury may act as a sink to exhaust the supply of plasma gelsolin, in turn allowing redundant inflammatory cascades unfettered opportunity to wreak havoc upon multiple organ systems.

The lower the levels of plasma gelsolin, the less favorable the prognosis of acute illness becomes [2-5,9]. Animal models further indicate that exogenous gelsolin administered during an identifiable window of opportunity can reduce injury and lower mortality [9-11]. More recently, evidence has begun to accumulate in chronic diseases. The current report by Osborn and colleagues provides data on patients with rheumatoid arthritis indicating that, at least in an acute-orchronic circumstance, circulating gelsolin concentrations are depressed below the normal range [1]. Further, the authors document the presence of gelsolin-actin complexes in synovial fluid. The identification of gelsolin-actin complexes inside the joint space is strong presumptive evidence of the sequestration hypothesis, as long as the isoform associated with the complexes is plasma gelsolin.

Rheumatoid arthritis represents a chronic inflammatory state punctuated by episodes of acute synovitis. Kulakowska and colleagues have recently demonstrated that blood and cerebrospinal fluid gelsolin concentrations in patients with multiple sclerosis are lower than levels for patients with peripheral neurological disorders [12]. In their patients, intrathecal actin was not detectable. The participants were all considered to be in the early stages of relapsing-remitting 
multiple sclerosis. Whether the chronic disease or an acute flare is responsible for lowering gelsolin levels can be hard to dissect in the setting of waxing and waning illness. Both aspects of such disease entities can increase consumption as well as restrain production of plasma proteins such as gelsolin.

Translational research in this area is also being optimistically pursued. Inhalational use of recombinant human gelsolin potentates the bactericidal activity of cationic endogenous antimicrobial peptides and exogenous antibiotics. In fact, gelsolin was first investigated as an inhaled therapeutic agent to decrease the viscosity of airway secretions from patients with cystic fibrosis. Appropriately dosed infusions of recombinant human gelsolin can diminish evolving injury or reduce mortality rates in animal models of hyperoxia, burns, and sepsis [9-11]. A particular advantage of gelsolin therapy over previously failed immunomodulary therapies derives from its modulation of multiple proinflammatory pathways. Gelsolin also inhibits certain actions of bacterial endotoxin. Gelsolin repletion to physiological levels between the time of the initial insult and subsequent complications in patients identified by marked hypogelsolinemia as being at substantial risk for delayed multiorgan dysfunction could provide a directed and effective therapeutic intervention in critically ill patients.

\section{Competing interests}

Although MJD currently has no financial connection to any company developing gelsolin for diagnostic or therapeutic uses, he did receive consulting fees more than 5 years ago from Biogen, which was studying the inhalational administration of recombinant human gelsolin to patients with cystic fibrosis. More recently, MJD has been an informal and unpaid advisor to Critical Biologics Corporation, which has acquired the rights to gelsolin. MJD is presently an employee of Merck \& Co., Inc., which has no current ties to gelsolin or Critical Biologics Corporation.

\section{Acknowledgement}

The author is indebted to Joann DiLullo for her indispensable assistance.

\section{References}

1. Osborn TM, Verdrengh M, Stossel TP, Tarkowski A, Bokarewa M: Decreased levels of the gelsolin plasma isoform in patients with rheumatoid arthritis. Arthritis Res Ther 2008, 10:R117.

2. Lee WM, Galbraith RM: The extracellular actin-scavenger system and actin toxicity. N Eng/ J Med 1992, 326:1335-1341.

3. Mounzer KC, Moncure M, Smith YR, DiNubile MJ: Relationship of admission plasma gelsolin levels to clinical outcomes in patients after major trauma. Am J Respir Crit Care Med 1999, 160:1673-1681.

4. DiNubile MJ, Stossel TP, Ljunghusen OC, Ferrara JL, Antin JH: Prognostic implications of declining plasma gelsolin levels after allogeneic stem cell transplantation. Blood 2002, 100: 4367-4371.

5. Lee PF, Drager LR, Stossel TP, Moore FD, Rogers SO: Relationship of plasma gelsolin levels in outcomes of critically ill surgical patients. Ann Surg 2006, 243:399-403.

6. Osborn TM, Dahlgren C, Hartwig JH, Stossel TP: Modifications of cellular responses to lysophosphatidic acid and platelet activating factor by plasma gelsolin. Am J Physiol Cell Physiol 2007, 292:C1323-C1330.
7. Bucki R, Georges PC, Espinassous Q, Funaki M, Pastore JJ, Chaby R, Janmey PA: Inactivation of endotoxin by human plasma gelsolin. Biochemistry 2005, 44:9590-9597.

8. Bucki R, Byfield FJ, Kulakowska A, McCormick ME, Drozdowski W, Bucki R, Namiot Z, Hartung T, Janmey PA: Extracellular gelsolin binds lipoteichoic acid and modulates cellular response to proinflammatory bacterial wall components. I Immumol 2008, 181:4936-4944.

9. Lee PS, Waxman AB, Cotich KL, Chung SW, Perrella MA, Stossel TP: Plasma gelsolin is a marker and therapeutic agent in animal sepsis. Crit Care Med 2007, 35:849-855.

10. Christofidou-Solomidou C, Scherpereel A, Solomides CC, Christie JD, Stossel TP, Goetz S, DiNubile MJ: Recombinant plasma gelsolin diminishes the acute inflammatory response to hyperoxia in mice. J Investig Med 2002, 50:54-60.

11. Rothenbach PA, Dahl B, Schwartz JJ, O'Keefe GE, Yamamoto M, Lee MW, Horton JW, Yin HL, Turnage RH: Recombinant plasma gelsolin infusion attenuates burn-induced pulmonary microvascular dysfunction. J Appl Physio/ 2004, 96:25-31.

12. Kulakowska A, Drozdowski W, Sadzynski A, Bucki R, Janmey PA: Gelsolin concentration in cerebrospinal fluid from patients with multiple sclerosis and other neurological disorders. Eur $J$ Neurol 2008, 15:584-588. 